\title{
Implications of multiply-charged anomalous cosmic rays
}

\author{
R. A. Mewaldt \\ California Institute of Technology, Pasadena, CA 91125
}

\begin{abstract}
During the acceleration of anomalous cosmic rays (ACRs) some singly-charged ions are stripped of additional electrons. The resulting multiply-charged ions are accelerated more efficiently, and most ACRs with $\geq 30 \mathrm{MeV} /$ nuc are multiply-charged. This paper considers some implications of multiply-charged ACRs, including limits that they place on the time scales for ACR acceleration and transport, and their use in defining the cutoff in the accelerated ACR spectrum. Possible causes of the spectral cutoff in three ACR acceleration models are discussed.
\end{abstract}

Keywords: Anomalous cosmic rays, termination shock, particle acceleration

PACS: $96.50 . \mathrm{Pw}, 96.50 . \mathrm{S}-$, 96.50.sb, 96.50.sh, 96.50.Ek

\section{INTRODUCTION}

Anomalous cosmic rays (ACRs) originate from interstellar neutral atoms that drift into the heliosphere, are ionized by solar UV or charge exchange with the solar wind, and then convected into the outer heliosphere where they are accelerated to energies of tens of MeV/nuc [1]. Pesses, Jokipii, and Eichler [2] first proposed that ACRs are accelerated at the solar-wind termination shock (TS) by diffusive shock acceleration. Indeed, ACR energy spectra at $1 \mathrm{AU}$ and in the outer heliosphere are consistent with those expected from shock acceleration models (e.g., [3]). Although Voyager 1 (V1) did not observe the expected shock-accelerated power-law spectrum in unmodulated form when it crossed the TS [4], we assume here that ACRs are accelerated at another location on the shock (e.g., [5]). Voyager-1 did find a weak shock with a compression ratio of $\sim 2.5[6]$.

The pickup-ion origin implies that ACRs should be singly-ionized [1], and several studies have shown that this is true of most ACRs with $10 \mathrm{MeV} /$ nuc (e.g., [7, 8, 9]). However, using data from the Solar, Anomalous, and Magnetospheric Explorer (SAMPEX), Mewaldt et al. [10] found that at energies $\geq 25 \mathrm{MeV} /$ nuc most ACRs have an ionic charge $\mathrm{Q} \geq 2$, as shown in Figure 1a. They interpreted this as evidence that some ACRs are stripped while being accelerated, and that ACRs gain energy in proportion to their ionic charge during acceleration as suggested by Jokipii [12]. This picture explains how some ACR oxygen are accelerated to energies as high as $\sim 100$ $\mathrm{MeV} /$ nuc $[13,10,14]$. The observed fraction ( 20\%) of multiply-charged to singlycharged $\mathrm{O}$ implies that ACRs are accelerated to $10 \mathrm{MeV} /$ nuc within 1-2 years $[10,14]$.

Further SAMPEX measurements showed that the $\mathrm{Q}=1 / \mathrm{Q} \geq 1$ ratio for $\mathrm{N}, \mathrm{O}$, and $\mathrm{Ne}$ crosses the $50 \%$ point at a common total energy of $\sim 360 \mathrm{MeV}$ (see Figure 1b). Using new stripping cross sections [18] and including stripping on neutral He and $\mathrm{O}$ [19], the

CP 858, Physics of the Inner Heliosheath,

edited by J. Heerikhuisen, V. Florinski, G. P. Zank, and N. V. Pogorelov

(C) 2006 American Institute of Physics 978-0-7354-0355-0/06/\$23.00 
cutoffs in the singly-charged spectra were well fit by the Jokipii model ([15], see Figure 1b). Other modelers have also considered multiply-charged ACRs. Steenberg [20] (see also [21]) found solutions similar to those of Jokipii [15]. In this model the $\mathrm{Q}=1 / \mathrm{Q} \geq 1$ ratio for $\mathrm{O}$ crosses $50 \%$ at $\sim 10 \mathrm{MeV} /$ nuc. Although Steenberg did not optimize the model to fit charge state data, he demonstrated that the cutoff energy for $\mathrm{O}$ occurs where the radial diffusion coefficient $\mathrm{K}_{\mathrm{RR}}=\mathrm{VR}_{\mathrm{S}} / 5.4$ (here $\mathrm{V}$ is solar wind speed and $R_{S}$ is the TS radius). He attributed the cutoff to the finite radius of curvature of the TS.
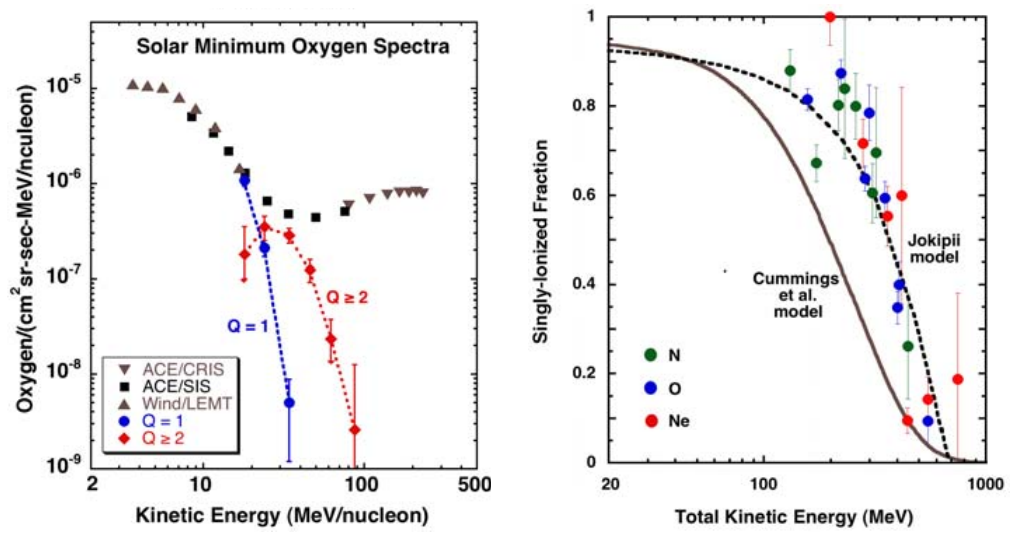

FIGURE 1: (a) Solar-minimum O spectra measured by ACE and Wind are shown along with the spectra of $\mathrm{Q}=1$ and $\mathrm{Q} \geq 2$ ions measured by SAMPEX using the geomagnetic technique [10, 11]. (b) The singly-charged oxygen fraction calculated by Jokipii [15] and CSS [5, 16] is compared to $\mathrm{N}, \mathrm{O}$, and Ne charge state measurements from SAMPEX $[9,17]$.

Cummings, Stone, and Steenberg ([3]; here-in-after CSS), using the same model, fit the solar-minimum spectra of six ACR species ( $\mathrm{H}, \mathrm{He}, \mathrm{N}, \mathrm{O}, \mathrm{Ne}$, and $\mathrm{Ar}$ ) at Earth, V1, and V2 (1, 70 and $~ 50$ AU), using both strong and weak termination shocks $(\mathrm{s}=4$ and $\mathrm{s}=2.4)$. They identified radial, latitudinal, and rigidity-dependent variations in the diffusion coefficient that could fit data from all three spacecraft. They also calculated the production of multiply-charged ACRs but did not publish details of the charge state distributions. Figure $1 \mathrm{~b}$ illustrates that in this calculation the $\mathrm{Q}=1 / \mathrm{Q} \geq 1$ ratio falls below $50 \%$ at $195 \mathrm{MeV}(12.2 \mathrm{MeV} /$ nuc). Table 1 summarizes cutoffs determined from $\mathrm{O}$ charge-state spectra, and from the location where the $\mathrm{O}$ spectrum drops to $50 \%$ of the extrapolated low-energy power law. Table 1 also includes other parameters to be discussed later. The differences between the cutoff energies for these models raise the question of what governs the location of these spectral cutoffs. In this paper we consider several possibilities.

TABLE 1. ACR Acceleration and Transport Models

\begin{tabular}{lccccc}
\hline Model & $\begin{array}{c}\mathbf{O}^{+} / \mathbf{O} \\
\text { Cutoff } \\
\text { (MeV/nuc) }\end{array}$ & $\begin{array}{c}\text { Power-law } \\
\text { Cutoff } \\
\text { (MeV/nuc) }\end{array}$ & $\begin{array}{c}\text { Accel. Time } \\
\text { to } \mathbf{1 0 ~ M e V / n u c} \\
\text { (years) }\end{array}$ & $\begin{array}{c}\mathbf{K}_{\mathrm{RR}} /\left(\mathbf{V R}_{\mathbf{S}} \text { ) at }\right. \\
\text { cutoff energy } \\
\text { (upstream) }\end{array}$ & $\begin{array}{c}\text { TS to HP } \\
\text { Diffusion } \\
\text { (years) }\end{array}$ \\
\hline Jokipii [15] & 22.4 & $\sim 12$ & $0.9-1.8$ & 0.82 & 2.6 \\
Steenberg [20,21] & $\sim 10$ & 4 & $1.0-2.4$ & 0.18 & 0.9 \\
CSS [5] & 12.2 & 6.4 & $0.09-0.21$ & 0.50 & 29 \\
\hline
\end{tabular}




\section{ACR SPECTRAL CUTOFFS}

To investigate cutoffs in ACR spectra it is important to consider the radial diffusion coefficients $\left(K_{R R}\right)$ and related scattering mean-free-paths $\left(\lambda_{R R}=3 K_{R R} / v\right.$, where $v$ is particle velocity) that are used to model ACR acceleration and transport. Figure 2 shows the radial dependence of $\lambda_{\mathrm{RR}}$ used by the three models considered above. There are several possible reasons why shock-accelerated particles could have a cutoff in the power-law spectrum, including 1) the acceleration time, 2) the radius of curvature of the shock, 3) particle escape (e.g., through the HP), and 4) energy-loss processes.

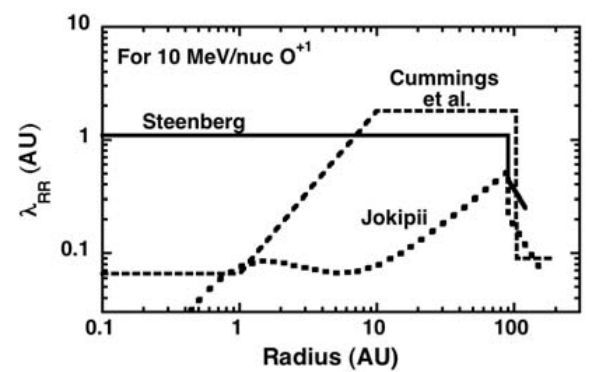

FIGURE 2: Radial mean free paths at $0^{\circ}$ latitude (for $10 \mathrm{MeV} /$ nuc $^{+1}$ ) in the ACR acceleration and transport models by Jokipii [15], Steenberg [20], and the weak-shock model of CSS [5].

Jokipii [22] finds that the acceleration time scale at perpendicular shocks like the termination shock is given by $\mathrm{T}_{\mathrm{AC}}=3 \mathrm{sK}_{\mathrm{RR}} /\left(\mathrm{V}^{2}(\mathrm{~s}-1)\right)$, where $\mathrm{K}_{\mathrm{RR}}$ is evaluated at the termination shock. Table 1 includes the time to accelerate oxygen to $10 \mathrm{MeV} /$ nuc for the three models (the range in values reflects latitude variations in $\mathrm{V}$ and $\mathrm{K}_{\mathrm{RR}}$ ). Note that the cutoff energies in the 3 models are not ordered by the acceleration times.

Particle acceleration becomes less efficient when the diffusive length scale $\left(\mathrm{K}_{\mathrm{RR}} / \mathrm{V}\right)$ becomes comparable to the shock radius $\left(\mathrm{K}_{\mathrm{RR}} / \mathrm{V} \sim 2 \mathrm{R}_{\mathrm{S}}\right)$ [23, 21]. Steenberg [20] and Steenberg and Moraal [21] attributed the spectral breaks in their model to shock-radius effects, but their cutoff occurred at a lower energy $\left(\sim 4 \mathrm{MeV} /\right.$ nuc, where $\mathrm{K}_{\mathrm{RR}} / \mathrm{V} \sim$ $0.09 \mathrm{R}_{\mathrm{S}}$ ) than the $\sim 46 \mathrm{MeV} /$ nuc value expected from $\mathrm{K}_{\mathrm{RR}} / \mathrm{V} \sim 2 \mathrm{R}_{\mathrm{S}}$ [23]. Table 1 includes the quantity $\mathrm{K}_{\mathrm{RR}} /\left(\mathrm{R}_{\mathrm{S}} \mathrm{V}\right)$ evaluated upstream, in the ecliptic, at the energy of the power-law cutoff for each model. Note that $\mathrm{K}_{\mathrm{RR}} /\left(\mathrm{R}_{\mathrm{S}} \mathrm{V}\right)$ is less than 2 to a variable degree, suggesting that shock-radius effects are not causing the cutoff in every case.

Similarly, the loss of particles through the HP may be significant if the time required for particles to diffuse to the HP is comparable to the acceleration time. Table 1 includes the estimated time for $10 \mathrm{MeV} /$ nuc $\mathrm{O}$ to diffuse from the TS to the $\mathrm{HP}$ [using $\mathrm{T}_{\text {dif }}=\left(\mathrm{R}_{\mathrm{HP}}-\mathrm{R}_{\mathrm{S}}\right)^{2} /\left(\mathrm{K}_{\mathrm{RR}}\right)$ ]. Of these models, this effect is most significant for the Steenberg [20] model, where the diffusion time to the HP is $\sim 9$ months. For the Jokipii and CSS models the TS to HP diffusion times are $\sim 2.6$ and $\sim 29$ years, respectively, and this effect should be negligible. ACRs can also be lost if they get far enough downstream that they miss the TS in diffusing back upstream. Florinski and Jokipii [24] concluded that this effect is negligible unless the HP radius, $R_{H}$, is $>R_{S}$, which is not the case in these models.

Florinski and Jokipii ([24], here-in-after F\&J) considered several effects on the spectra of particles accelerated at spherical termination shocks. Using radial diffusion 
coefficients of the form $K_{R R}=K_{1}\left(P / P_{o}\right)^{\alpha}\left(R / R_{S}\right)$, where $P$ is momentum, they considered solutions for "small diffusion" and "large diffusion" limits, which apply if $\left(\mathrm{VR}_{\mathrm{S}} / \mathrm{K}_{1}\right)\left(\mathrm{P} / \mathrm{P}_{\mathrm{o}}\right)^{-\alpha}$ is $>>1$ or $<<1$ upstream of the shock. In either case the usual power-law spectrum from shock acceleration has an exponential cutoff, such that:

$$
\mathrm{f}(\mathrm{P}) \sim \mathrm{P}^{-\sigma} \exp \left[\left(-\mathrm{bK} \mathrm{K}_{1} / \mathrm{VR}_{\mathrm{S}}\right)\left(\mathrm{P} / \mathrm{P}_{\mathrm{o}}\right)^{\alpha}\right]
$$

The small-diffusion approximation of F\&J [24] is more appropriate here, in which $\mathrm{b}=2 \sigma(\sigma-\alpha-3) /\left(3 \alpha \mathrm{VR}_{\mathrm{S}}\right)$, with $\sigma=3 \mathrm{~s} /(\mathrm{s}-1)$. Then, if the downstream $\mathrm{K}_{\mathrm{RR}}<<\mathrm{K}_{\mathrm{RR}}$ upstream (limiting particle loss through the HP), F\&J found that the primary loss mechanism is due to adiabatic cooling upstream of the TS. Physically, what happens is that as particles gain energy they can penetrate more easily into the inner heliosphere where they lose energy at a faster rate $\left[\mathrm{T}_{\mathrm{ad}} \sim 3 \mathrm{R} /(2 \mathrm{~V})\right]$. When they do return to the shock, it is at a lower energy, which effectively limits the number of particles accelerated to high energy and causes the spectrum to steepen.
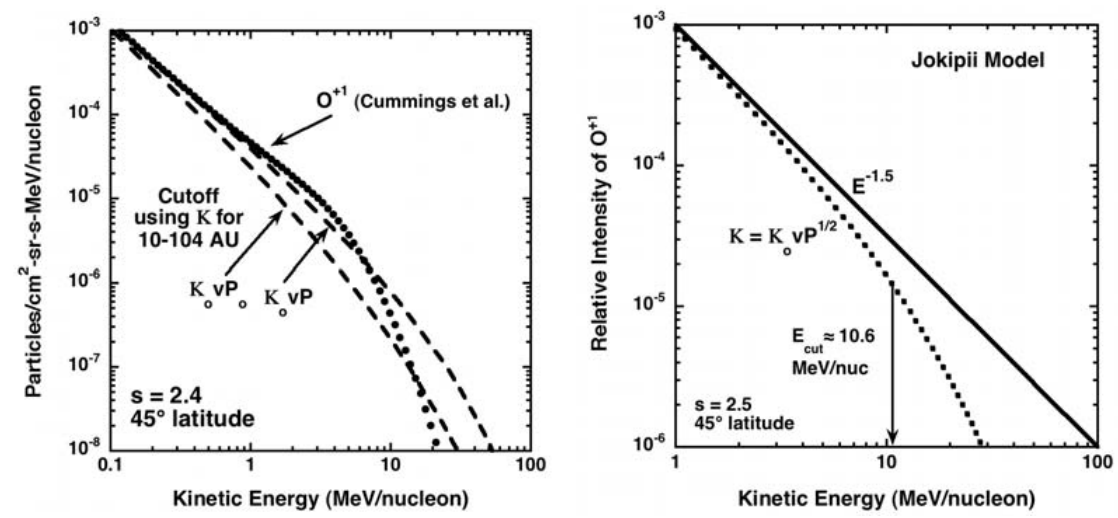

FIGURE 3: (a) The cutoff in the CSS oxygen spectrum is compared with estimates based on the small diffusion model of F\&J. (b) Cutoff estimate for the Jokipii model $\left(45^{\circ}\right.$ latitude).

To see if the F\&J solutions can reproduce the cutoff in the CSS ACR spectra we need to accommodate the more complex radial dependence of $\mathrm{K}_{\mathrm{RR}}$ (Figure 2) into the $\mathrm{K}_{\mathrm{RR}}$ proportional to $\mathrm{R}$ form assumed by $\mathrm{F} \& \mathrm{~J}$. We selected $\mathrm{K} 1$ so that the integral of $\mathrm{K}_{1}\left(\mathrm{R} / \mathrm{R}_{\mathrm{S}}\right)^{2}$ equaled the integral of the CSS $\mathrm{K}_{\mathrm{RR}}$ over the volume from $0.1 \mathrm{AU}$ to $\mathrm{R}_{\mathrm{S}}$. To accommodate the dependence of $\mathrm{K}_{\mathrm{RR}}$ on rigidity (corresponding to $\alpha=1.5$ for $0.45 \mathrm{GV}$ to $1.4 \mathrm{GV}$ and to $\alpha=1$ for $>1.4 \mathrm{GV}$, we tried solutions with both $\alpha=1$ and $\alpha=1.5$ (see Figure 3a). For $\alpha=1$ the cutoff in the spectrum (point where it falls $50 \%$ below the power law) is at $\sim 1.1 \mathrm{MeV} /$ nuc, while for $\alpha=1.5$ the cutoff is at $8.6 \mathrm{MeV} /$ nuc. The actual $\mathrm{O}$ cutoff in the CSS model is between these approximations, at $6.4 \mathrm{MeV} /$ nuc. As a second check, the cutoff in the Jokipii (2000) oxygen spectrum is estimated to be $10.6 \mathrm{MeV} /$ nuc using the $\mathrm{F} \& \mathrm{~J}$ approximation (Figure $3 \mathrm{~b}$ ), in reasonable agreement with the value in Table 1 . Here the radial and rigidity dependences of $\lambda$ are consistent with the assumptions of F\&J. In both cases the shape of the exponential cutoff in this approximation is more gradual than in the models themselves. 


\section{ACR TIME SCALES}

The fraction of multiply-charged ACRs (e.g., the $\mathrm{O}^{+1} / \mathrm{O}$ ratio) provides a measure of the age of ACRs since they were accelerated and/or the distance they have traveled. For mean free paths that are independent of rigidity $\left(\mathrm{K}_{\mathrm{RR}} \sim \mathrm{K}_{\mathrm{o}} \mathrm{V}\right)$ the time to accelerate to a given energy is proportional to $\mathrm{E}^{0.5}$, while for $\mathrm{K}_{\mathrm{RR}}=\mathrm{K}_{\mathrm{o}} \mathrm{vP}$ the acceleration time is proportional to energy. Figure $4 \mathrm{a}$ shows the $\mathrm{O}^{+1} / \mathrm{O}$ fraction versus the time to accelerate to $10 \mathrm{MeV} /$ nuc for these cases. The ACR spectra observed by V1 beyond the TS have led to suggestions that ACRs may be accelerated in locations other than the TS, including the heliosheath. If this is so, the acceleration time to $10 \mathrm{MeV} / \mathrm{nuc}$ must be 1-2 years or less.
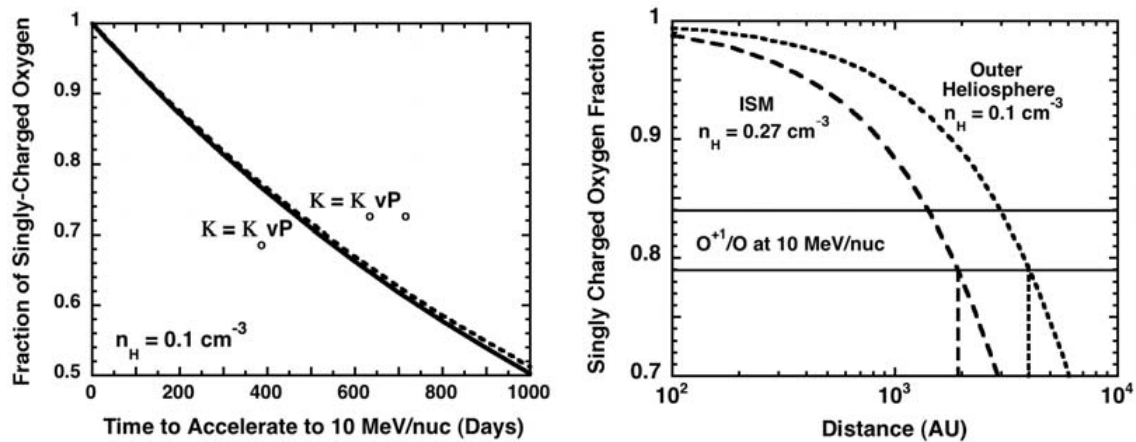

FIGURE 4 (a) The $\mathrm{O}^{+} / \mathrm{O}$ ratio is plotted versus the time to accelerate singly-charged ions from $\sim 0.01$ to $10 \mathrm{MeV} /$ nuc. The acceleration rates are for $\mathrm{K}_{\mathrm{RR}}=\mathrm{K}_{\mathrm{o}} \mathrm{vP} \mathrm{O}_{\mathrm{O}}\left(\mathrm{T}_{\mathrm{ac}}\right.$ proportional to $\left.\mathrm{v}\right)$ and $\mathrm{K}_{\mathrm{RR}}=\mathrm{K}_{\mathrm{o}} \mathrm{vP}$ ( $\mathrm{T}_{\mathrm{ac}}$ proportional to $\mathrm{E}$ ). (b) The $\mathrm{O}^{+} / \mathrm{O}$ ratio at $10 \mathrm{MeV} /$ nuc is shown versus the distance traveled from the acceleration site (neglecting stripping during acceleration) for acceleration sites inside and outside the HP $\left(\mathrm{n}_{\mathrm{H}}=0.1 \mathrm{~cm}^{-3}\right.$ and $0.27 \mathrm{~cm}^{-3}$, respectively). These illustrative calculations are for a beam of particles that start out together; they are not based on a complete, steady-state acceleration/transport model that includes other energy-loss processes.

It is also possible that ACRs are accelerated beyond the termination shock and then transported into the inner heliosphere. Based on a mean charge state for $\sim 10 \mathrm{MeV} / \mathrm{nuc}$ ACR oxygen of $\mathrm{Q}<1.8$, Adams and Leising [25] concluded that ACRs originate less than $0.2 \mathrm{pc}$ from Earth. With better charge state measurements and improved cross sections, this limit can be improved on, as shown in Figure 4b. We conclude that ACRs have traveled $<4000 \mathrm{AU}$ from their acceleration site if they originate inside the $\mathrm{HP}$, and $<2000 \mathrm{AU}$ if they originate in the interstellar medium (ISM).

\section{DISCUSSION}

There are several effects that can lead to spectral cutoffs, and it is possible that the limiting parameter is not the same in the models discussed here. The CSS model [3] has the fastest acceleration, an intermediate value of $\mathrm{K}_{\mathrm{RR}} /\left(\mathrm{R}_{\mathrm{S}} \mathrm{V}\right)$, provides the least access to the HP (based on Figure 2; see Table 1), but provides the easiest access to the inner heliosphere (see Figure 2). Since $\mathrm{T}_{\mathrm{AC}}$ is so small, and downstream losses are minimized, it is clear that adiabatic cooling upstream of the shock must be the main cause of the cutoff. The Steenberg model [20] has the lowest cutoff energy, the 
slowest acceleration rate, the smallest value of $\mathrm{K}_{\mathrm{RR}} /\left(\mathrm{R}_{\mathrm{S}} \mathrm{V}\right)$, and allows the easiest access to the HP (see Figure 2 and Table 1). Adiabatic cooling in the inner heliosphere must be important, but the cutoff is most likely less than that for CSS because of much easier particle escape through the heliopause. The Jokipii model [16] has the highest cutoff energy, but the other parameters in Table 1 lie between those of the other two models. Given that the F\&J model provides a reasonable estimate of the cutoff, we suggest that the main reason for the higher cutoff energy is that shock-accelerated particles have less access to the inner heliosphere in this model (Figure 2). Detailed simulations (e.g., [24]) could test these conclusions.

If a significant part of the acceleration of ACRs takes place at locations other than the termination shock, or by mechanisms other than diffusive shock acceleration, neither the acceleration time scale or the matter traversed can be so great as to overproduce multiply-charged ACRs. Thus, ACR charge states can provide a key test of alternate acceleration sites and mechanisms.

\section{ACKNOWLEDGMENTS}

I appreciate very much the use of unpublished charge-state calculations for the CSS model provided by A. C. Cummings. I also acknowledge helpful discussions with A. C. Cummings, E. C. Stone, V. Florinski, J. R. Jokipii, H. Moraal, and C. D. Steenberg. This work was supported by NASA under grants NAG5-12929 and NAG5-8877.

\section{REFERENCES}

1. L. A. Fisk, B. Koslovsky and R. Ramaty, Ap. J. Letters, 190, L35-L38 (1974).

2. M. E. Pesses, J. R. Jokipii and D. Eichler, Ap. J. Letters, 246, L85-L88 (1981).

3. A. C. Cummings, E. C. Stone, and C. D. Steenberg, Ap. J. 578, 194-210 (2002).

4. E. C. Stone, A. C. Cummings, F. B. McDonald et al., Science, 309, 2017-2020 (2005).

5. D. J. McComas and N. A. Schwadron, Geophys. Res. Lett. 33, (4), DOI 10.1029/2005GL025437.

6. L. F. Burlaga et al., Science, 309, 2027 (2005).

7. A. C. Cummings, E. C. Stone, and W. R. Webber, Ap. J. Letters, 287, L99-L102 (1984)

8. J. H. Adams, Jr., M. Garcia-Munoz, and N. L. Grigorov et al., Ap. J. Letters 375, L45-L48 (1991).

9. B. Klecker, M. C. McNab, J. B. Blake, et al., Ap. J. Letters 442, L69-L72 (1995).

10. R. A. Mewaldt, R. S. Selesnick, J. R. Cummings, and E. C. Stone, Ap. J. Letters 466, L43-L46 (1996).

11. R. A. Leske, R. A. Mewaldt, et al., in Acceleration and Transport of Energetic Particles Observed in the Heliosphere, R. A. Mewaldt et al., eds., AIP Conf. Proc. \#528 AIP, Melville, NY, 2000, pp. 293-300.

12. J. R. Jokipii, in Physics of the Outer Heliosphere, S. Grzedzielski and D. E. Page, eds, Pergamon Press (1990).

13. R. A. Mewaldt, J. R. Cummings, R. A. Leske, et al., Geophys. Res. Letters, 23, 617-620 (1996).

14. J. R. Jokipii, Ap. J. Letters 466, L47-L50 (1996).

15. J. R. Jokipii, in Acceleration and Transport of Energetic Particles Observed in the Heliosphere, edited by R. A. Mewaldt et al., AIP Conference Proceedings 528 AIP, Melville, NY, 2000, pp. 309-316.

16. A. C. Cummings, private communication, (2006).

17. B. Klecker, R. A. Mewaldt, and M. Oetliker et al., Space Science Reviews, 83, 294-299 (1998).

18. A. F. Barghouty, Phys. Rev. A, 61, 052702-1-052702-3 (2000).

19. A. F. Barghouty, J. R. Jokipii, and R. A. Mewaldt, in The Outer Heliosphere: The Next Frontiers, K. Scherer et al. eds, COSPAR Colloquia Series Vol. 11, Pergamon, (2001), pp. 203-206.

20. C. D. Steenberg, in Acceleration and Transport of Energetic Particles Observed in the Heliosphere, R. A. Mewaldt et al., eds., AIP Conference Proceedings 528 AIP, Melville, NY, 2000, pp. 301-308.

21. C. D. Steenberg and H. Moraal, J. Geophys. Res. 104, 24879-24884 (1999).

22. J. R. Jokipii, Ap. J. Letters, 393, L41-L43 (1992).

23. L. O'C Drury, Rep. Prog. Phys. 46, 973-1027 (1983).

24. V. Florinski and J. R. Jokipii, Ap. J. 591, 454-460 (2003).

25. J. H. Adams, Jr. and M. D. Leising, Proc. $22^{\text {nd }}$ Internat. Cosmic Ray Conf., 3, 304-307 (1991). 